Discrete Comput Geom 28:571-575 (2002)

DOI: $10.1007 / \mathrm{s} 00454-002-2887-0$

\title{
Restricted Point Configurations with Many Collinear $k$-Tuplets
}

Dan Ismailescu

Department of Mathematics, Hofstra University,

Hempstead, NY 11549-1000, USA

matdpi@hofstra.edu

Abstract. Given $k \geq 3$, denote by $t_{k}^{\prime}(N)$ the largest integer for which there is a set of $N$ points in the plane, no $k+1$ of them on a line such that there are $t_{k}^{\prime}(N)$ lines, each containing exactly $k$ of the points. Erdós (1962) raised the problem of estimating the order of magnitude of $t_{k}^{\prime}(N)$. We prove that

$$
t_{k}^{\prime}(N) \geq \begin{cases}c_{k}^{\prime} N^{\log (k+4) / \log (k)} & \text { if } \quad 4 \leq k \leq 17, \\ c_{k}^{\prime} N^{1+1 /(k-3.59)} & \text { if } \quad k \geq 18\end{cases}
$$

improving a previous bound of Grünbaum for all $k \geq 5$. The proof for $k \geq 18$ uses an argument of Brass with his permission.

\section{Introduction}

Denote by $t_{k}(N)$ the largest integer for which there is a set of $N$ points in the plane such that there are $t_{k}(N)$ lines each containing exactly $k$ of the points. The particular case $k=3$ (also known as "the orchard problem") has been studied for more than 150 years. It is known that $t_{3}(N)=N^{2} / 6+O(N)$; for the sharpest results on $t_{3}(N)$ (as well as for the history of the problem and many interesting references) the reader is referred to the paper of Burr et al. [2].

Croft and Erdős (see [8] or the survey paper of Erdős and Purdy [7]) showed that for every $k \geq 3$,

$$
t_{k}(N) \geq \frac{c}{k^{3}} N^{2},
$$

where $c$ is an absolute constant. Szemerédi and Trotter [11] and later (with a completely different approach and a much better constant) Clarkson et al. [3] proved that this result is the best possible; however, the best value of $c$ is still not known. 
Erdős [4] considered the following variant of the above problem: for each $k \geq 3$ determine $t_{k}^{\prime}(N)$, the largest integer for which there is a set of $N$ points in the plane, no $k+1$ of them on a line, such that there are $t_{k}^{\prime}(N)$ lines, each containing exactly $k$ of the points.

Using iterated Minkowski sums, Kárteszi [10] proved that $t_{k}^{\prime}(N) \geq c_{k} N \log N$ for all $k \geq 3$. Projecting the lattice cube $\{1, \ldots, k\}^{n}$ on the plane gives a set of $N=k^{n}$ points, no $k+1$ of them on a line, containing $\left((k+2)^{n}-k^{n}\right) / 2$ collinear $k$-tuples, which implies the better bound $t_{k}^{\prime}(N) \geq c_{k} N^{\log (k+2) / \log (k)}$.

The best lower bound is due to Grünbaum [9], who used a recursive technique (which we describe later in this paper) to show that

$$
t_{k}^{\prime}(N) \geq c_{k} N^{1+1 /(k-2)} \quad \text { for all } \quad k \geq 3 .
$$

The improved bounds mentioned in the abstract will be obtained by combining a new construction presented below with Grünbaum's argument.

\section{The New Construction}

In this section we present a point-configuration with no $k+1$ points on a line and "many" collinear $k$-tuples.

For any positive integer $k \geq 3$, denote by $A_{k}$ the following set of $k$ consecutive integers:

$$
A_{k}:=\left\{i \text { integer } \mid\left[1-\frac{k}{2}\right] \leq i \leq\left[\frac{k}{2}\right]\right\}, \quad \text { where }[x] \text { denotes the greatest integer } \leq x .
$$

Given a positive integer $n$, let $H_{k, n}$ be defined as

$$
H_{k, n}:=\left\{\mathbf{v} \in A_{k}{ }^{n} \mid \text { the first nonzero component of } \mathbf{v} \text { is equal to }-1\right\} .
$$

It is easy to see that $\left|H_{k, n}\right|=\left(k^{n}-1\right) /(k-1)$.

Lemma. The set $B(k, n):=\bigcup_{i=1}^{k-1}(1 / i) H_{k, n}$ has the following properties:

1. $|B(k, n)|=k^{n}-1$.

2. No $k+1$ points of $B(k, n)$ lie on the same line.

3. There are at least $\left(\left((k+4)^{n}-1\right) /(k+3)-\left(k^{n}-1\right) /(k-1)\right) / 2$ collinear $k$-tuples determined by points of $B(k, n)$.

Proof. 1. This is easy to see since each of the sets $(1 / i) H_{k, n}$ has exactly $\left(k^{n}-1\right) /(k-1)$ points; moreover, from our choice of $H_{k, n}$ it is clear that for $i, j$ distinct between 1 and $k-1$, the corresponding sets $(1 / i) H_{k, n}$ and $(1 / j) H_{k, n}$ are disjoint.

2. Suppose that there exist $k+1$ collinear points in $B(k, n)$. Then, for some particular $1 \leq p \leq k-1,(1 / p) H_{k, n}$ will contain at least two of these points, denote them by $\mathbf{u}$ and $\mathbf{v}$. Let $\mathbf{w}$ be another point of $B(k, n)$, on the line determined by $\mathbf{u}$ and $\mathbf{v}$. Then we can write $\mathbf{w}=\lambda \mathbf{u}+(1-\lambda) \mathbf{v}$ for some real $\lambda \neq 0,1$. 
We now distinguish two cases:

2a: $\mathbf{u}$ and $\mathbf{v}$ have the first nonzero component in the same position. Then the first nonzero component of $\mathbf{w}$ is $\lambda(-1 / p)+(1-\lambda)(-1 / p)=-1 / p$, that is $\mathbf{w} \in(1 / p) H_{k, n}$. In other words, in this case every point belonging to the line determined by $\mathbf{u}$ and $\mathbf{v}$ should be in $(1 / p) H_{k, n}$, too. However, no more than $k$ points of $(1 / p) H_{k, n}$ can lie on the same line.

$2 \mathrm{~b}: \mathbf{u}$ and $\mathbf{v}$ do not have the first nonzero component in the same position. With no loss of generality we can assume that the first nonzero component of $\mathbf{u}$ appears in an earlier position than the first nonzero component of $\mathbf{v}$. In this case the first nonzero component of $\mathbf{w}$ is $\lambda \cdot(-1 / p)$. On the other hand, since $\mathbf{w} \in B(k, n)$ it follows that the first nonzero component of $\mathbf{w}$ is $-1 / q$ for some $1 \leq q \leq k-1$. Therefore, $-1 / q=\lambda \cdot(-1 / p)$, that is, $\lambda=p / q$. Since $\lambda \neq 1$ it follows that $q \neq p$ which leaves us with at most $k-2$ choices for $q$. Now, $\mathbf{w}=(p / q) \mathbf{u}+(1-p / q) \mathbf{v}$ which means that there are at most $k-2$ other points in $B(k, n)$ on the line determined by $\mathbf{u}$ and $\mathbf{v}$. Hence, there can be no $k+1$ collinear points in this case either.

3. We will estimate the number of pairs $\mathbf{u}, \mathbf{v} \in H_{k, n}$ for which $(\mathbf{u}+j \mathbf{v}) /(1+j) \in$ $(1 /(1+j)) H_{k, n}$ for all $1 \leq j \leq k-2$. Clearly, every such point lies on the line segment determined by $\mathbf{u}$ and $\mathbf{v}$ and therefore, for different pairs $\mathbf{u}, \mathbf{v}$, we obtain distinct $k$-tuplets of collinear points from $B(k, n)$.

Equivalently, we want to count the number of pairs $\mathbf{u}, \mathbf{v} \in H_{k, n}$ for which $\mathbf{u}+j \mathbf{v} \in H_{k, n}$ for all $1 \leq j \leq k-2$. Suppose now that the first nonzero component of $\mathbf{u}$ is on the $(m+1)$ st position and the first nonzero component of $\mathbf{v}$ is on the $(m+p+2)$ nd position, where $0 \leq m \leq n-2$ and $0 \leq p \leq n-m-2$ (see Fig. 1). In other words, we consider only those pairs $\mathbf{u}, \mathbf{v}$ for which the first nonzero component of $\mathbf{u}$ appears in an earlier position than that of $\mathbf{v}$. The $(m+2)$ nd up to the $(m+p+1)$ st components of $\mathbf{u}$ can be chosen arbitrarily from $A_{k}=\{[1-k / 2], \ldots,[k / 2]\}$ since the corresponding components of $\mathbf{v}$ are all equal to 0 . The $(m+p+2)$ nd component of $\mathbf{u}$ can be chosen to be either $[k / 2]-1$ or $[k / 2]$.

For every $i \in A(k)$ we denote by $s_{i}$ the number of occurrences of $i$ among the last $(n-m-p-2)$ components of $\mathbf{u}$. For every occurrence of [1-k/2] or [2-k/2] among these last $(n-m-p-2)$ components of $\mathbf{u}$, the corresponding component of $\mathbf{v}$ can be chosen to be either 0 or 1 . Similarly, for every occurrence of $[k / 2-1]$ or $[k / 2]$ among the last $(n-m-p-2)$ components of $\mathbf{u}$, the corresponding component of $\mathbf{v}$ can be chosen to be either 0 or -1 . For all the other values of the last $n-m-p-2$ components of $\mathbf{u}$ in $A(k)$, the correspondent coordinate of $\mathbf{v}$ should be necessarily 0 , in order to have $\mathbf{u}+j \mathbf{v} \in H_{k, n}$ for all $1 \leq j \leq k-2$.

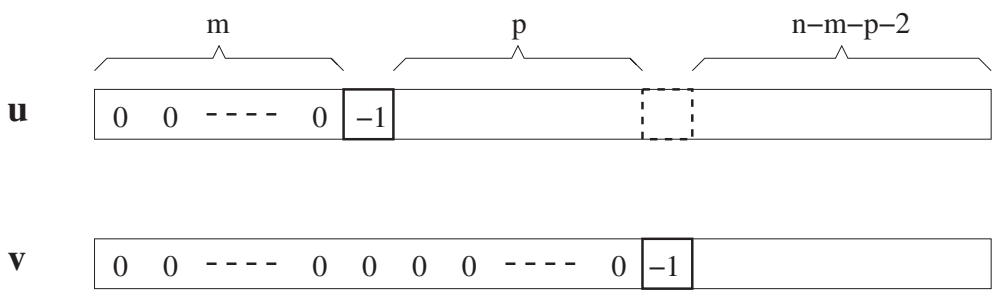

Fig. 1. Choosing $\mathbf{u}$ and $\mathbf{v}$ so that $\mathbf{u}+j \mathbf{v} \in H_{k, n}$. 
Hence, the number $P$ of pairs $\mathbf{u}, \mathbf{v}$ satisfying the above conditions is given by

$$
P=\sum_{m=0}^{n-2} \sum_{p=0}^{n-m-2} k^{p} \cdot 2 \cdot \sum\left(\begin{array}{c}
n-m-p-2 \\
s_{[1-k / 2]}, \ldots, s_{[k / 2]}
\end{array}\right) \cdot 2^{s_{[1-k / 2]}} \cdot 2^{s_{[2-k / 2]}} \cdot 2^{s_{[k / 2-1]}} \cdot 2^{s_{[k / 2]}},
$$

where the interior sum is taken over all nonnegative integer solutions of the equation $\sum_{i \in A(k)} s_{i}=n-m-p-2$. It follows that

$$
P=\sum_{m=0}^{n-2} \sum_{p=0}^{n-m-2} k^{p} \cdot 2 \cdot(k+4)^{n-m-p-2}
$$

and after some straightforward computations

$$
P=\left(\frac{(k+4)^{n}-1}{k+3}-\frac{k^{n}-1}{k-1}\right) / 2 .
$$

Observation. An appropriate planar projection of the set $B(k, n)$ has $k^{n}-1$ points, no $k+1$ of which are collinear, while the number of collinear $k$-tuples is at least $(((k+$ $\left.\left.4)^{n}-1\right) /(k+3)-\left(k^{n}-1\right) /(k-1)\right) / 2$. Therefore,

\section{Theorem 1.}

$$
t_{k}^{\prime}(N) \geq c_{k}^{\prime} N^{\log (k+4) / \log (k)} \quad \text { for all } \quad k \geq 3 .
$$

The above bound gives the same order $\Omega\left(n^{3 / 2}\right)$ as (1) for $k=4$ and it is better for $5 \leq k \leq 35$.

\section{Better Bounds for $k \geq 18$}

Brass [1] recently noticed that the above result can be used in conjunction with Grünbaum's original argument in order to obtain an improvement of (1) for all values of $k \geq 5$. We include the short proof for the sake of completeness.

Theorem 2 [1], [9].

$$
t_{k}^{\prime}(N) \geq c_{k}^{\prime} N^{1+1 /(k-3.59)} \quad \text { for all } \quad k \geq 18
$$

The proof (of the projectively dual assertion) is by induction on $k$. For $k=18$ the inequality follows from Theorem 1. Suppose we have an arrangement $A(n, k)$ of $n$ lines that determine $d=c_{k}^{\prime} n^{1+1 /(k-3.59)} k$-tuple points (vertices) $P_{1}, P_{2}, \ldots, P_{d}$, but no point is on more than $k$ lines.

We show that $t_{k+1}^{\prime}(N) \geq c_{k+1}^{\prime} N^{1+1 /(k+1-3.59)}$ as follows:

In the Euclidean 3-space we take one copy of $A(n, k)$ in the plane $z=1$, and another copy of $A(n, k)$ in the plane $z=q$, for a suitable integer $q$, the second copy rotated by an angle $\alpha$ with respect to the first. Let $l_{1}, l_{2}, \ldots, l_{d}$ be the straight lines connecting corresponding vertices in the two copies. For each $b=2,3, \ldots, q-1$, the $d$ intersection 
points of the plane $z=b$ with the lines $l_{1}, l_{2}, \ldots, l_{d}$ form a set which is linearly equivalent to the set $P_{1}, P_{2}, \ldots, P_{d}$, and therefore lies on $n$ suitable lines.

Thus we have a family of $N=q n+d$ lines that determine $q d$ vertices of multiplicity $k+1$ and, for a suitable choice of $\alpha$, no vertex of greater multiplicity. A suitable parallel projection of these lines yields a planar arrangement of lines with the same property. Choosing now $q$ to be about $n^{1 /(k-3.59)}$, it follows that $N$ is about $\left(1+c_{k}^{\prime}\right) \cdot n^{1+1 /(k-3.59)}$. Therefore,

$$
t_{k+1}^{\prime}(N)=t_{k+1}^{\prime}(q n+d) \geq q d=c_{k}^{\prime} n^{1+2 /(k-3.59)}=c_{k+1}^{\prime} N^{1+1 /(k+1-3.59)}
$$

and the proof is completed.

\section{Conclusions and Open Problems}

The problem of estimating the true order of magnitude of $t_{k}^{\prime}(N)$ for $k \geq 4$ remains wide open. There are no subquadratic upper bounds. For example, $t_{4}^{\prime}(N) \leq \frac{1}{12} N^{2}$ is trivial, and only recently Brass [1] proved that $t_{4}^{\prime}(N) \leq \frac{1}{14} N^{2}+O(N)$.

In his paper [9], Grünbaum says: "it is well possible that our result may be improved to $t_{k}^{\prime}(N) \geq c_{k}^{*} N^{2}$." On the other hand, Erdős (see, e.g., [5] and [6]), repeatedly conjectured that $t_{k}^{\prime}(N)=o\left(N^{2}\right)$, for all $k \geq 4$.

The most interesting case remains $k=4$, the only value of $k$ for which we could not improve Grünbaum's lower bound.

We hope that this paper will attract some attention to this interesting and difficult problem which seems to have been undeservedly forgotten.

\section{References}

1. P. Brass. On point sets without $k$ collinear points. Manuscript, 2001.

2. S. A. Burr, B. Grünbaum, and N. J. A. Sloane. The orchard problem. Geom. Dedicata., 2:397-424, 1974.

3. K. L. Clarkson, H. Edelsbrunner, L. J. Guibas, M. Sharir, and E. Welzl. Combinatorial complexity bounds for arrangements of curves and spheres. Discrete Comput. Geom., 5(2)-99-160, 1990.

4. P. Erdôs. Néhány elemi geometriai problémárol. Köz. Mat. Lapok, 24:193-201, 1962.

5. P. Erdős. Some combinational problems in geometry. In Geometry and Differential Geometry (Proc. Conf., Univ. Haifa), pages 46-53. Number 792 in Lecture Notes in Mathematics. Springer-Verlag, Berlin, 1979.

6. P. Erdős. Problems and results in combinatorial geometry. In Discrete Geometry and Convexity, pages 1-11. Number 440 in Annals of the New York Academy of Science. New York Academy of Science, New York, 1985.

7. P. Erdős and G. Purdy. Extremal problems in combinatorial geometry. In Handbook of Combinatorics, number 48, pages 809-874. Elsevier, Amsterdam, 1995.

8. B. Grünbaum. Arrangements and Spreads. Volume 10 of CBMS Regional Conference Series in Mathematics. Conference Board of Mathematical Sciences, Washington, DC, 1972.

9. B. Grünbaum. New views on some old questions of combinatorial geometry. In Colloquio Internazionale sulle Teorie Combinatorie (Rome, 1973), Tomo I, pp. 451-468. Atti dei Convegni Lincei, No. 17. Accademia Nazionale dei Lincei, Rome, 1976.

10. F. Kárteszi. Sylvester egy tétéléröl és Erdôs egy sejtéséröl. Köz. Mat. Lapok, 26:3-10, 1963.

11. E. Szemerédi and W. T. Trotter. Extremal problems in discrete geometry. Combinatorica, 3:381-392, 1983.

Received February 21, 2001, and in revised form December 15, 2001. Online publication October 29, 2002. 